\title{
Clavicle nonunion and plate breakage after locking compression plate fixation of displaced midshaft clavicular fractures
}

\author{
XIAOYAN HUANG, HAIJUN XIAO and FENG XUE \\ Department of Orthopedics, Shanghai Fengxian District Central Hospital, Shanghai 201400, P.R. China
}

Received November 15, 2018; Accepted November 11, 2019

DOI: $10.3892 /$ etm.2019.8216

\begin{abstract}
Open reduction and plate fixation have been widely used for the treatment of displaced midshaft clavicular fractures (DMCF). The nonunion rate after plate fixation of DMCF has been reported to be between 0.1 and $15 \%$ and the construct failure rate is approximately $5 \%$. Few studies have discussed the risk factors of construct failure. The aim of the present study was to identify possible risk factors of construct failure in plate fixation of DMCF and discuss the subsequent treatment strategies. Six patients who experienced plate breakage and clavicle nonunion between 2015 and 2017 were evaluated. All these patients were treated with open reduction and plate fixation of DMCF using a 3.5-mm locking compression plate. The plate breakage occurred 3-6 months after the initial injury. After the diagnosis of plate breakage, four patients underwent surgical management and two patients underwent nonoperative treatment. Potential risk factors for construct failure and efficacy of the subsequent treatment strategies were analyzed. We found that a risk factor for plate breakage was the increased stress in free hole area around the fracture zone. A second surgery for plate renewal and bone grafting may be necessary in a large percentage of these individuals. Based on the results of this study, our recommendation is that monocortical screws or simple obturators for the holes around the fracture zone should be used to protect the comminuted fragment for further damage and enhance plate strength. If a clavicle nonunion and plate breakage does occur, surgical repair and bone grafting provide high union rates and should be a necessary remedy.
\end{abstract}

\section{Introduction}

Clavicular fracture is quite common accounting for approximately $5 \%$ of all fractures in adults. More than $80 \%$ of these

Correspondence to: Professor Haijun Xiao or Professor Feng Xue, Department of Orthopedics, Shanghai Fengxian District Central Hospital, 6600 Nanfeng Road, Fengxian, Shanghai 201400,P.R. China E-mail: xiaohaijun89@126.com

E-mail: fxgkxf@sina.com

Key words: clavicle nonunion, plate breakage, locking compression plate, displaced midshaft clavicular fractures fractures are located in the middle third of the clavicle and are displaced $(1,2)$. Several clinical trials have shown better functional outcomes after open reduction and internal fixation for displaced midshaft clavicular fractures (DMCF), and have confirmed the superiority of operative treatment in clinical practice $(3,4)$. Additionally, the nonunion rate seems to be lower after operative treatment (0-3\%) than conservative treatment $(21 \%)(4,5)$. Recent studies have shown that construct failure rates range from 1.2 to $12.6 \%$, including breaking or bending of the plate and screw loosening (6-9).

Plates can be subdivided into reconstruction plates and locking compression plates. Reconstruction plates have a lower profile with a concentrated mass around the screw holes which reduces the plate stiffness, resulting in a higher failure rate than the locking compression plate when used for the fixation of displaced clavicular fractures (6). Locking compression plates, available in a straight and anatomically pre-shaped design, are stronger and therefore much more difficult to bend (6). The failure rate of locking compression plate is low, and there is little literature discussing the associated risk factors. Additionally, there are few studies reporting whether second surgery or nonoperative treatment was required in the occurrence of plate breakage with fracture nonunion.

The primary aim of this study was to identify possible risk factors for construct failure after the locking compression plate fixation of DMCF. The secondary aim of this study was to discuss the treatment strategies after the diagnosis of plate breakage and fracture nonunion.

\section{Materials and methods}

This is a retrospective study, based on medical records from the archives of the Shanghai Fengxian District Central Hospital, from 2015 to 2017. This clinical study was approved by the Shanghai Fengxian District Central Hospital Medical Ethics Committee, and informed consent was obtained from the patients for surgical procedures and inclusion of data in the study.

Six patients diagnosed with fracture nonunion and plate breakage were included in this study. Preoperative demographic and clinical variables are documented, including age of the patient at the time of injury, gender, laterality, hand dominance, mechanism of injury, treatment of the fracture preceding the nonunion, and treatment of the nonunion after the diagnosis of plate breakage. More specific nonunion risk 
factors were also investigated, including prior clavicle fractures, previous clavicle surgery, smoking history, and diabetes.

Plain X-ray film or computerized tomography (CT) scans were carried out immediately after the injury, 2 days after the surgery, and at every follow-up.

\section{Results}

Six patients were included in the study. The demographic data of the patients are documented in Table I. The fracture nonunion and plate breakage occurred between 3 and 6 months after the first surgery. All patients reported no history of injury before the plate breakage but did have a history of daily heavy lifting. The breakage site was near the free hole area around the fracture zone. After the diagnosis of clavicle nonunion and plate breakage, four patients received revision surgery of plate renewal and bone grafting, and two patients received nonoperative treatment of figure-of-eight splint and sling immobilization. All of the four patients with revision surgery realized fracture union 3-9 months after the second surgery (Figs. 1 and 2). One patient with nonoperative treatment still could not realize fracture union and possibly needed revision surgery in the near fracture (Fig. 3). The other patient with nonoperative treatment realized partial fracture union 6 months after the diagnosis of plate breakage (Fig. 4).

\section{Discussion}

The clavicle is the major supporting structure for the shoulder, and is mainly positioned under two frequent loading modes: bending and compressive loads (10). Clavicle fractures are the most frequent cited fractures of the shoulder with most of them are located in the midshaft; this is related to the morphological and mechanical features of the bone where the two inverse curves enable the bone to absorb stress (11). Open reduction and plate fixation are the common routine methods for the treatment of clavicle fractures.

Reconstruction plates have a lower profile with a concentrated mass around the screw holes which reduces the plate stiffness. Locking compression plates, available in a straight and anatomically pre-shaped design, and precisely pre-contoured to reduce the need for additional intraoperative contouring, are stronger and much more difficult to bend. Gilde et al (12) discouraged the use of reconstruction plates because of the higher rate of plate failure in comparison to the stiffer dynamic compression plate. Currently, locking compression plates are the mainstream internal implant for clavicle fracture. Yet, locking compression plates still display a certain rate of fixation failure.

When discussing implant failure, mechanical and biological factors should be taken into account (10). The biological factor is usually related to poor bone quality; when the failure point is located at the bone-screw interface, the biological factor may play a more important role. The mechanical factor usually concerns bending stress on the bone that is transmitted to the implant and generates a failure located at the screw-plate junction. We suspected that our case was mainly due to a complex type of mechanical factors because the patients are all middle-aged and have a low rate of osteoporosis.

The traditional AO principles regarding bridge plating $(6,13)$ recommend to leave at least two or three plate
Table I. Demographics and technical data of the patients in the present study $(n=6)$.

Demographic and technical data

No. of patients

Sex

Male

6

Female

0

Age, mean (range) in years

46.7 (35-68)

Mechanism of injury

Fall from height

4

Road traffic accident

2

Laterality

Left

1

Right

5

Hand dominance

Left

0

Right

6

Prior clavicle fractures

Yes

0

No

Prior clavicle surgery

Yes

0

No

6

Smoking history

Yes

3

No

3

Diabetes history

Yes

0

No

6

AO classification

Completely displaced midshaft fracture

4

Partially displaced midshaft fracture

2

Length of first stay (days)

$7 \pm 1$

Length between initial surgery and

3-6

plate breakage (months)

History of injury before plate breakage

Yes

No

6

holes without screws in the fracture zone to avoid stress concentration and plate failure. But in this case, we found that plate breakage usually occurred between 3 and 6 months after the first surgery, and the breakage point was right in the free holes area around the fracture zone. Because of the fracture pattern, it was not possible for a screw insertion in the intermediary bone fragment without the risk for further damage. Therefore, the unobstructed holes in this bridging technique created a zone of minimal resistance in the area free of screws, which induced plate breakage. It may be hypothesized that an appropriate plate should not have unobstructed holes at all, and monocortical screws or obturators placed in the free holes may enhance mechanical plate integrity and prevent its failure. 
A

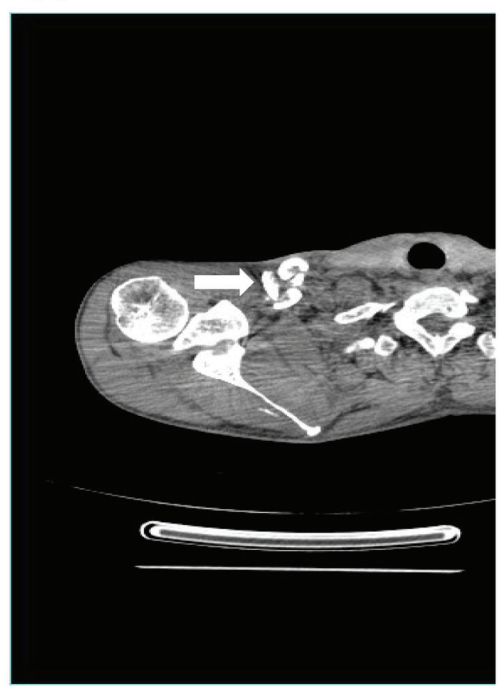

D

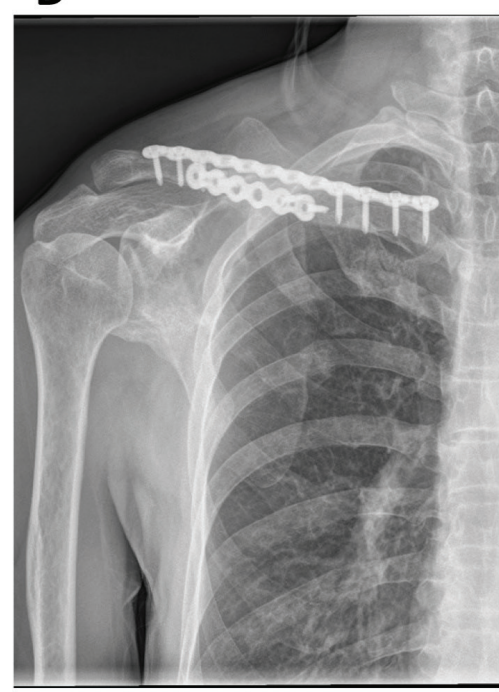

B

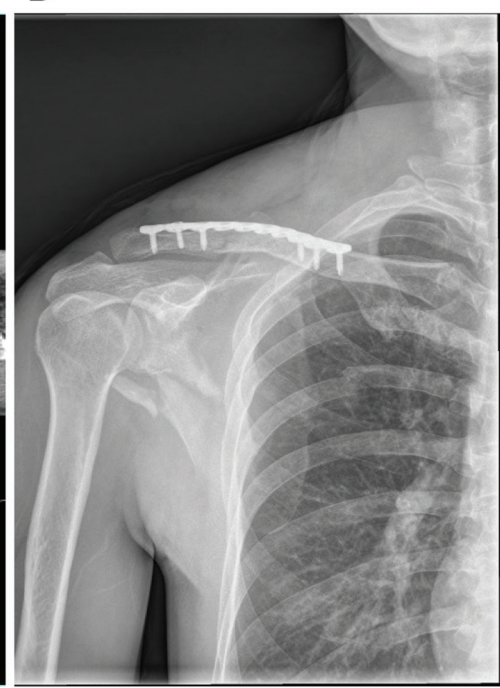

E
C

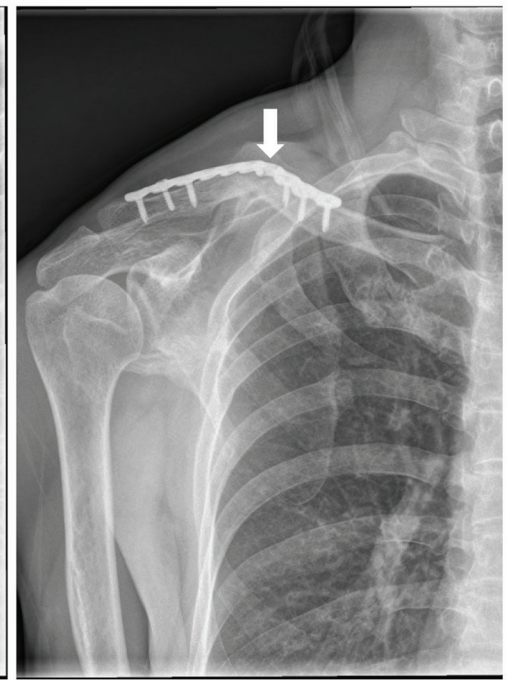

$\mathbf{F}$

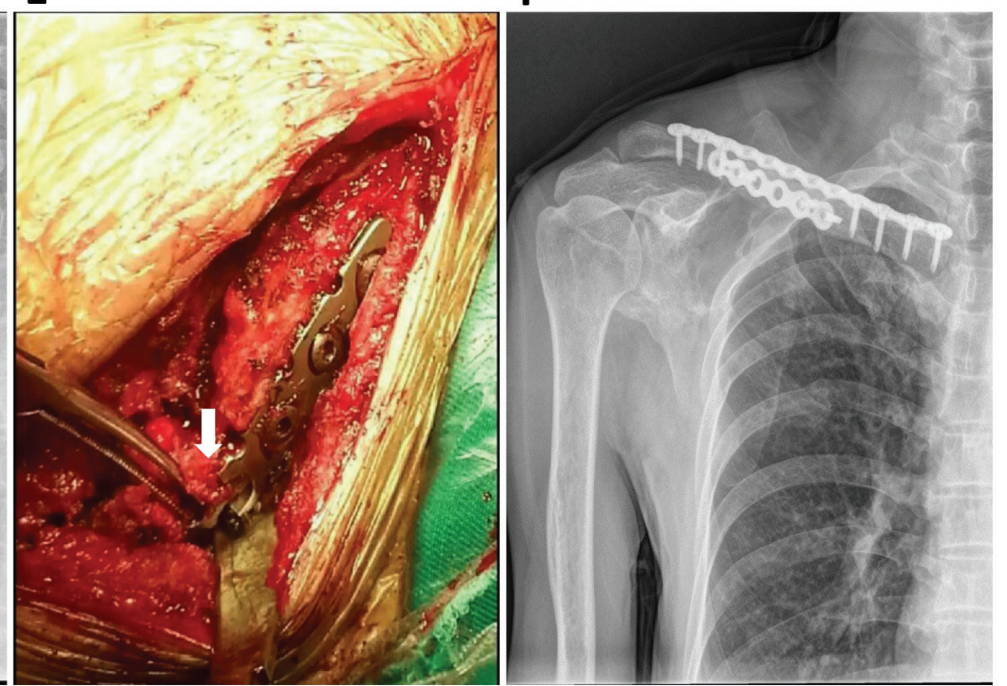

Figure 1. A case with plate breakage and clavicle nonunion after open surgery was treated with plate renewal and bone grafting. (A) CT scanning of the right clavicle in the emergency department showing the clavicle fracture zone (white arrow). (B) X-ray AP view of the right clavicle 2 days after open surgery showing good fracture reduction. (C) X-ray AP view of the right clavicle 5 months after open surgery showing clavicle nonunion and plate breakage (white arrow). (D) X-ray AP view of the right clavicle 2 days after the renewal of plate and bone grafting (white arrow. (E) Intraoperative image showed the plate breakage point was at the free hole area around the fracture zone. (F) X-ray AP view of the right clavicle 3 months after the revision surgery showing union of the fracture. AP, anteroposterior.

A

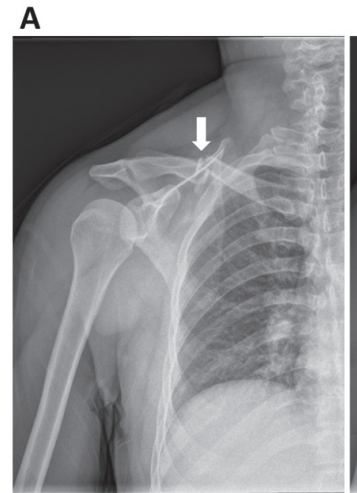

B

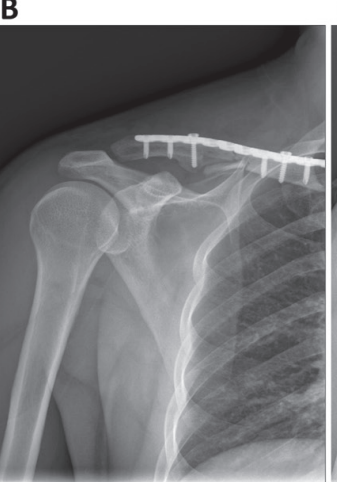

C

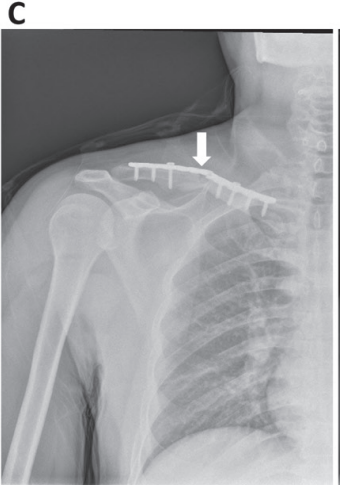

D

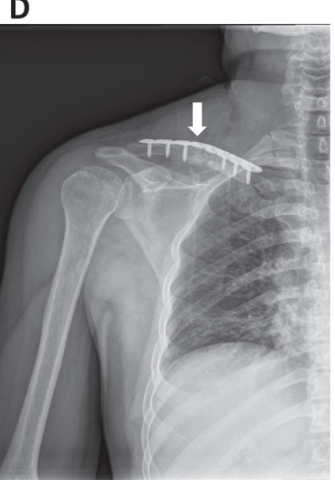

E

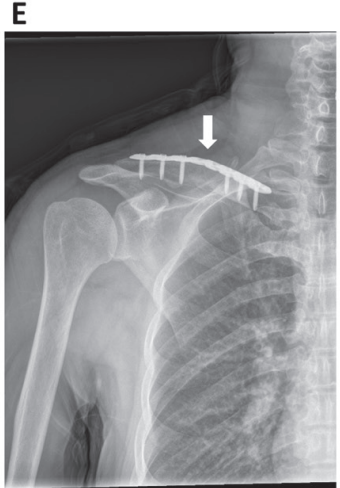

Figure 2. A case with plate breakage and clavicle nonunion after open surgery was treated with plate renewal. (A) X-ray AP view of the right clavicle in the emergency department showing the clavicle fracture zone (white arrow). (B) X-ray AP view of the right clavicle 2 days after open surgery showing the good fracture reduction. (C) X-ray AP view of the right clavicle 5 months after open surgery showed the clavicle nonunion and plate breakage (white arrow). (D) X-ray AP view of the right clavicle 2 days after renewal of the plate (white arrow). (E) X-ray AP view of the right clavicle 3 months after the revision surgery showing union of the fracture (white arrow). AP, anteroposterior. 
A

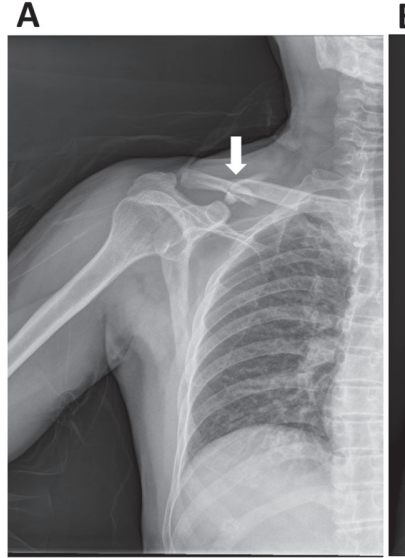

B

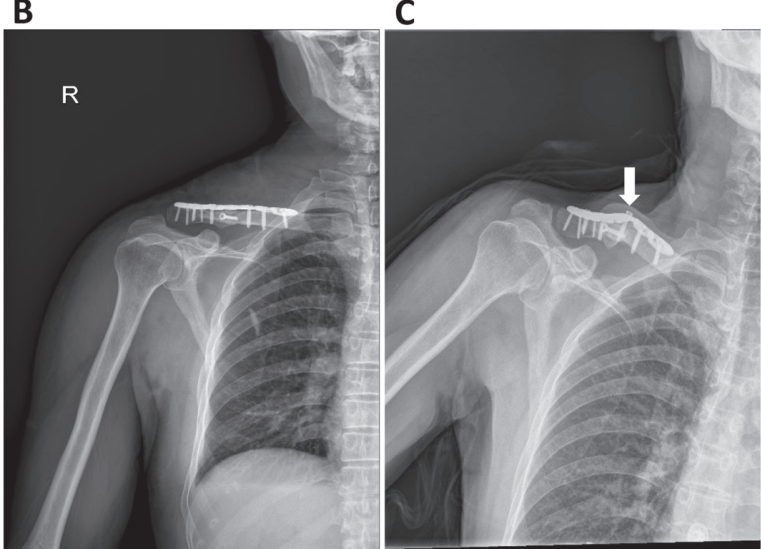

D

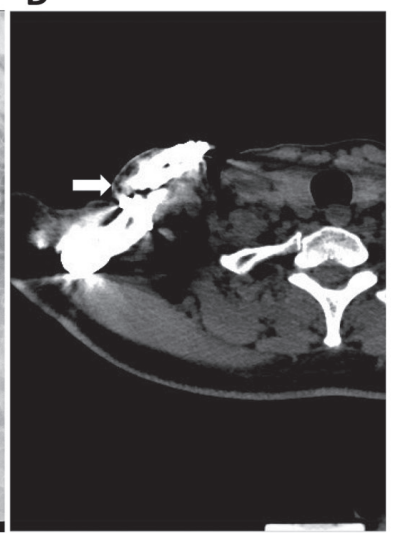

Figure 3. A case with plate breakage and clavicle nonunion after open surgery was treated with nonoperative management. (A) X-ray AP view of the right clavicle in the emergency department showing the clavicle fracture zone (white arrow). (B) X-ray AP view of the right clavicle 2 days after open surgery showing the good fracture reduction. (C) X-ray AP view of the right clavicle 6 months after open surgery showing the clavicle nonunion and plate breakage (white arrow). (D) CT scan of the right clavicle 12 months after the open surgery showing little union of the fracture (white arrow), and the possible need for revision surgery in the near future. AP, anteroposterior.

A

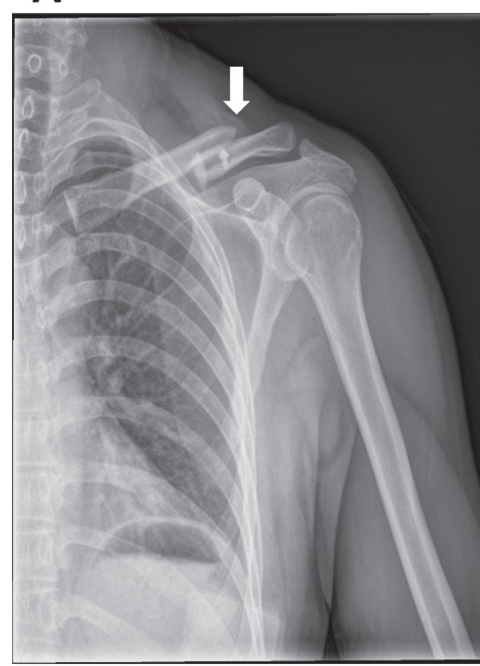

D

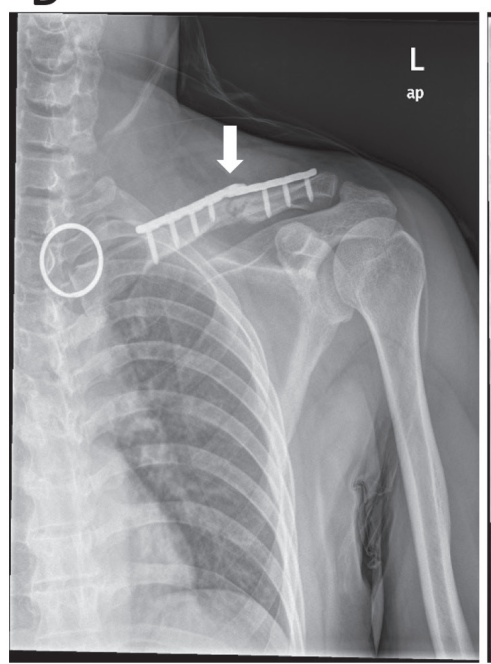

B

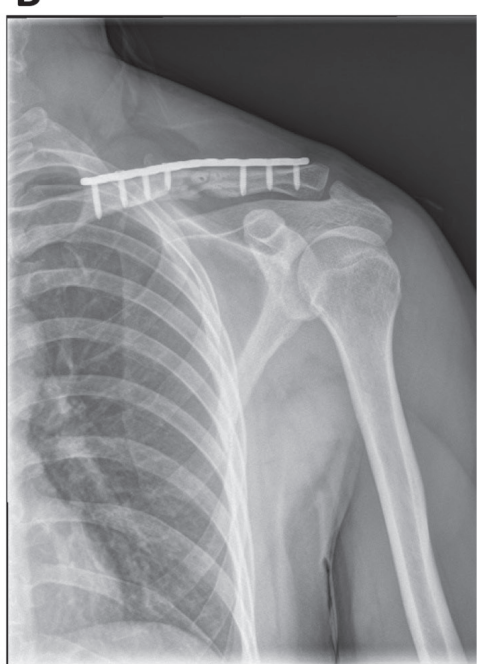

E

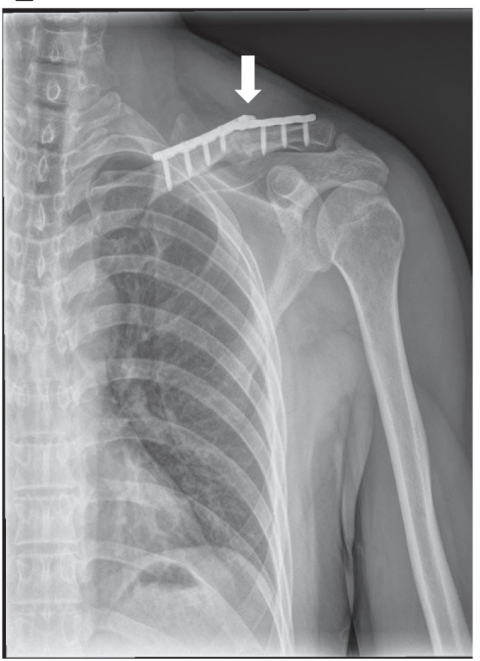

C

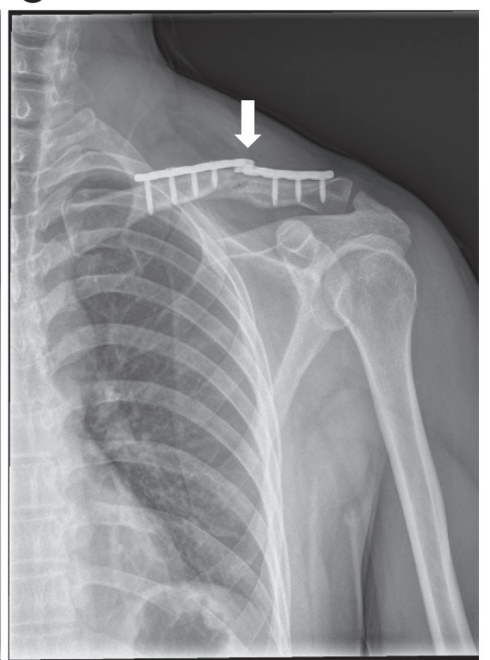

$\mathbf{F}$

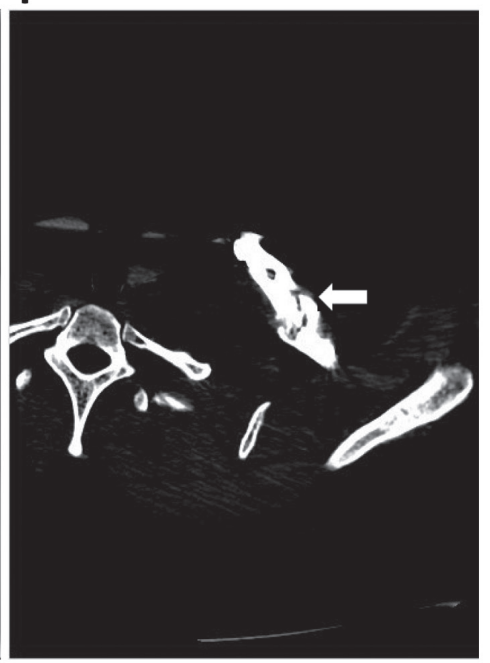

Figure 4. A case with plate breakage and clavicle nonunion after open surgery was treated with nonoperative management. (A) X-ray AP view of the right clavicle in the emergency department showing the clavicle fracture zone (white arrow). (B) X-ray AP view of the right clavicle 2 days after open surgery showing good fracture reduction. (C) X-ray AP view of the right clavicle 4 months after open surgery showing the clavicle nonunion and plate breakage (white arrow). (D) X-ray AP view of the right clavicle 6 months after open surgery showing the clavicle nonunion and plate breakage (white arrow). (E) X-ray AP view of the right clavicle 9 months after open surgery (white arrow). (F) CT scan of the right clavicle 12 months after open surgery showed partial fracture union (white arrow). AP, anteroposterior. 
Previous studies have shown that surgical techniques of clavicular nonunion and plate breakage seem to be acceptable with a good clinical outcome $(6,10)$. In the majority of cases, the authors performed a corrective osteotomy followed by plate fixation and/or bone grafting. This seems to be the preferred technique in the literature, as the primary goal of surgical intervention should be the restoration of clavicle anatomy (especially length, rotation and alignment) while creating an environment that is conducive to bony union (14). In our case, the patients treated with plate renewal and bone grafting realized fracture union in the next 3-9 months. One patient treated with nonoperative management still did not realize fracture union and there was a high probability for this patient to receive surgical treatment in the near future. The other patient treated with nonoperative management realized partial fracture union after 7 months of diagnosis of the plate breakage. Thus, we believe that surgical treatment is necessary after the diagnosis of fracture nonunion and plate breakage. A rigid fixation by means of a plate to prevent rotation and allow early mobilization combined with bone grafting to create a conducive osteoblastic environment seems to be the best option for most patients with clavicle malunions.

There are still several limitations to the present study. Firstly, because of the small sample size, we could not perform biomechanics analysis to further support our hypothesis. Secondly, the retrospective design of the study also impaired its validity. Thirdly, we also did not use monocortical screws in the cases. Thus, this study is a case report. In the future, a higher number of cases must be collected and detailed biomechanic analyses must be performed to confirm our hypothesis.

In conclusion, it was demonstrated that the increase in stress in the free holes area around the fracture zone is a risk factor for fracture nonunion and plate breakage, and monocortical screws or simple obturators for the holes around the fracture zone should be used to protect the comminuted fragment for further damage and enhance plate strength. After the diagnosis of fracture nonunion and plate breakage, a second surgery for plate renewal and bone grafting could provide high union rates, and should be a necessary remedy.

\section{Acknowledgements}

Not applicable.

\section{Funding}

The present work was supported by the Natural Science Foundation of Fengxian District, Shanghai (grant no. 20181703) and by the Foundation of Shanghai Municipal Commission of Health and Family Planning (grant no. 201840135).

\section{Availability of data and materials}

The datasets used and/or analyzed during the current study are available from the corresponding author on reasonable request.

\section{Authors' contributions}

XH and HX researched and collected the data for the study. FX and HX designed and directed the research study. XH wrote the manuscript. All authors read and approved the manuscript and agree to be accountable for all aspects of the research in ensuring that the accuracy or integrity of any part of the work are appropriately investigated and resolved.

\section{Ethics approval and consent to participate}

The present study was approved by the Shanghai Fengxian District Central Hospital Medical Ethics Committee and written informed consent was obtained from all patients.

\section{Patient consent for publication}

All patients agreed with the publication of this article.

\section{Competing interests}

The authors declare that they have no competing interests.

\section{References}

1. Nordqvist A and Petersson C: The incidence of fractures of the clavicle. Clin Orthop Relat Res: 127-132, 1994.

2. Postacchini F, Gumina S, De Santis P and Albo F: Epidemiology of clavicle fractures. J Shoulder Elbow Surg 11: 452-456, 2002.

3. Bernstein J: Nonoperative treatment compared with plate fixation of displaced midshaft clavicular fractures. J Bone Joint Surg Am 89: 1866-1867, 2007.

4. Robinson CM, Goudie EB, Murray IR, Jenkins PJ, Ahktar MA, Read EO, Foster CJ, Clark K, Brooksbank AJ, Arthur A, et al: Open reduction and plate fixation versus nonoperative treatment for displaced midshaft clavicular fractures: A multicenter, randomized, controlled trial. J Bone Joint Surg Am 95: 1576-1584, 2013.

5. Robinson CM, Court-Brown CM, McQueen MM and Wakefield AE: Estimating the risk of nonunion following nonoperative treatment of a clavicular fracture. J Bone Joint Surg Am 86: 1359-1365, 2004.

6. Meeuwis MA, Pull Ter Gunne AF, Verhofstad MH and van der Heijden FH: Construct failure after open reduction and plate fixation of displaced midshaft clavicular fractures. Injury 48: 715-719, 2017.

7. Fridberg M, Ban I, Issa Z, Krasheninnikoff $M$ and Troelsen A: Locking plate osteosynthesis of clavicle fractures: Complication and reoperation rates in one hundred and five consecutive cases. Int Orthop 37: 689-692, 2013.

8. Andrade-Silva FB,KojimaKE, Joeris A, Santos Silva J and Mattar R Jr: Single, superiorly placed reconstruction plate compared with flexible intramedullary nailing for midshaft clavicular fractures: A prospective, randomized controlled trial. J Bone Joint Surg Am 97: 620-626, 2015.

9. Woltz S, Duijff JW,Hoogendoorn JM, Rhemrev SJ, Breederveld RS, Schipper IB and Beeres FJ: Reconstruction plates for midshaft clavicular fractures: A retrospective cohort study. Orthop Traumatol Surg Res 102: 25-29, 2016.

10. Marinescu R, Antoniac VI, Stoia DI and Lăptoiu DC: Clavicle anatomical osteosynthesis plate breakage-failure analysis report based on patient morphological parameters. Rom J Morphol Embryol 58: 593-598, 2017.

11. Bachoura A, Deane AS, Wise JN and Kamineni S: Clavicle morphometry revisited: A 3-dimensional study with relevance to operative fixation. J Shoulder Elbow Surg 22: e15-e21, 2013.

12. Gilde AK, Jones CB, Sietsema DL and Hoffmann MF: Does plate type influence the clinical outcomes and implant removal in midclavicular fractures fixed with 2.7-mm anteroinferior plates? A retrospective cohort study. J Orthop Surg Res 9: 55, 2014.

13. Wagner M: General principles for the clinical use of the LCP. Injury 34 (Suppl 2): B31-B42, 2003.

14. Martetschläger F, Gaskill TR and Millett PJ: Management of clavicle nonunion and malunion. J Shoulder Elbow Surg 22: 862-868, 2013.

This work is licensed under a Creative Commons Attribution-NonCommercial-NoDerivatives 4.0 International (CC BY-NC-ND 4.0) License. 